\title{
Flavor-changing neutral currents in 331 models
}

\author{
J M Cabarcas ${ }^{1}$, D Gómez Dumm ${ }^{1}$ and R Martinez ${ }^{2}$ \\ ${ }^{1}$ IFLP, CONICET-Dpto. de Física, Universidad Nacional de La Plata, C.C. 67, 1900 La Plata, \\ Argentina \\ ${ }^{2}$ Dpto. de Física, Universidad Nacional, Bogotá, Colombia \\ E-mail: dumm@ fisica.unlp.edu.ar (Daniel Gómez Dumm)
}

Received 30 October 2009

Published 11 February 2010

Online at stacks.iop.org/JPhysG/37/045001

\begin{abstract}
We carry out a general analysis of tree-level flavor-changing neutral currents in the context of 331 models, considering arbitrary quark and gauge boson mixing matrices. The results are applied to definite textures of quark mass matrices, and differences between various 331 scenarios are pointed out.
\end{abstract}

\section{Introduction}

It is well known that in the standard model (SM) flavor-changing neutral currents (FCNC) are forbidden at the tree level; thus, FCNC-mediated processes are only allowed through one or more loop corrections. On the experimental side, even if the sizes of FCNC in the $u-c, b-s$, $s-d$ and $b-d$ sectors are found to be in good agreement with SM predictions, there is still room available for the presence of new physics.

In this paper we address the problem of FCNC in the context of the so-called 331 models, in which the $\mathrm{SM}$ gauge group is enlarged to $S U(3)_{C} \otimes S U(3)_{L} \otimes U(1)_{X}$ [1]. This extension of the gauge group implies the presence of a new neutral gauge boson $Z^{\prime}$, which in general gives rise to tree-level FCNC. The existence of $Z^{\prime}$ gauge bosons occurs not only in 331 models but also in many popular extensions of the SM, and in general $Z^{\prime}$ searches are part of the main experimental programs in high-energy physics. Among the various extensions of the SM, 331 models have the attractive feature of addressing the problem of fermion family replications. The puzzle is partially solved through the requirement of anomaly cancellations [1-4], since in the 331 framework the number of quarks families turns out to be related to the number of colors. In addition, 331 models show a rich phenomenology, which includes the presence of new scalars and heavy quarks, and offers the possibility of see-saw mechanisms to generate neutrino masses [5], dark matter candidates [6], gauge coupling unification [7], etc.

It is important to emphasize that in 331 models it is not possible to fit all SM quark and lepton families in multiplets having the same quantum numbers. As a consequence, the corresponding $Z^{\prime}$ couplings are not universal for all fermion flavors, which leads to tree-level FCNC [8-14]. Moreover, the couplings of left-handed fermions to the 'standard' Z-like 
gauge boson also include tree-level FCNC, both through $Z-Z^{\prime}$ mixing and through the mixing between ordinary and exotic quarks (the latter, in 331 models where exotic quarks have ordinary electric charges). The sizes of the couplings depend on the angles and phases of the (left-handed) up- and down-quark mixing matrices $V_{L}^{u}$ and $V_{L}^{d}$, which become separately observable. It is noticeable that, because of the unitarity of these matrices, predictions for FCNC observables in 331 models are in general related to each other [11, 12, 15]. Moreover, the extensions of the SM fermion and scalar sectors lead to new CP violation sources when quark and/or lepton mixing are taken into account. Experimentally, the nonstandard neutral currents could be identified e.g. at the LHC by looking at the process $p p \rightarrow Z^{\prime} \rightarrow e^{+} e^{-}$: performing specific kinematic cuts on the outgoing electrons, it would be possible to reduce background so as to distinguish the $Z^{\prime}$ current within 331 models from other theories that include physics beyond the SM [16].

In this work we present a general analysis of the various sources of tree-level FCNC in 331 models, showing the relations between the involved quark mixing matrix elements. In particular, we consider the case in which nonstandard quarks carry ordinary $2 / 3$ and $-1 / 3$ electric charges; thus, they mix with ordinary fermions and lead to tree-level FCNC both through $Z$ - and $Z^{\prime}$-quark couplings. In this case one also finds further neutral heavy gauge bosons, which mix with the $Z$ and $Z^{\prime}$ states and may provide a source of $C P$ violation. The aim of this paper is to present an overall analysis of FCNC sources, generalizing previous results obtained in particular 331 theories. Our results are compared with model-independent studies that do not include Z-mediated FCNC [17, 18], and with previous analyses within 331 models in which exotic quarks have nonstandard electric charges, and therefore do not mix with ordinary fermions $[8,11,19]$. The mixing between $\mathrm{CP}$-even and $\mathrm{CP}$-odd neutral gauge bosons is also taken into account.

The paper is organized as follows: in section 2 we introduce the models under study. In section 3 we analyze the couplings between fermions and gauge bosons, focusing on the study of FCNC. The case in which fermion mixing matrices show family hierarchies is considered. An application of this formalism to analyze different contributions to neutral meson mass differences is presented in section 4. Finally, section 5 includes a brief summary of our results.

\section{331 models}

As stated above, in 331 models, the SM gauge group is enlarged to $S U(3)_{C} \otimes S U(3)_{L} \otimes U(1)_{X}$. Left-handed fermions are organized into $S U(3)_{L}$ triplets, which in general include the standard quarks and leptons, as well as new exotic particles. The criterion of anomaly cancellation leads to some constraints in the allowed fermion representations ( $\mathbf{3}$ or $\overline{\mathbf{3}}$ ) and the fermion quantum numbers.

To describe the particle content of the model, we start by defining the electric charge operator, which can be written as a linear combination of the diagonal generators of the group:

$$
Q=T_{3}+\beta T_{8}+X
$$

Here $\beta$ is a parameter that characterizes the specific particle structure and quantum numbers of the model. For arbitrary $\beta$ the quark content of the multiplets consistent with anomaly cancellation requirements and a minimal number of exotic particles [4] is as follows: 


$$
\begin{array}{rlrl}
\mathcal{Q}_{i L} & =\left(\begin{array}{lll}
d_{i} & -u_{i} & J_{i}
\end{array}\right)_{L}^{T} \sim\left(\overline{\mathbf{3}}, \frac{1}{6}+\frac{\beta}{2 \sqrt{3}}\right), & i=1,2 \\
\mathcal{Q}_{3 L} & =\left(\begin{array}{lll}
d_{3} & u_{3} & J_{3}
\end{array}\right)_{L}^{T} \sim\left(\mathbf{3}, \frac{1}{6}-\frac{\beta}{2 \sqrt{3}}\right) \\
u_{j R} & \sim\left(\mathbf{1}, \frac{2}{3}\right), \quad d_{j R} \sim\left(\mathbf{1},-\frac{1}{3}\right), \quad j=1,2,3 \\
J_{i R} & \sim\left(\mathbf{1}, \frac{1}{6}+\frac{\sqrt{3} \beta}{2}\right), \quad i=1,2, \quad J_{3 R} \sim\left(\mathbf{1}, \frac{1}{6}-\frac{\sqrt{3} \beta}{2}\right),
\end{array}
$$

where entries in the parentheses label the $S U(3)_{L}$ representation and the $X$ quantum number. It is easy to see that the electric charges of exotic quarks $J$ are given by $Q_{J_{1}}=Q_{J_{2}}=1 / 6+$ $\sqrt{3} \beta / 2$ and $Q_{J_{3}}=1 / 6-\sqrt{3} \beta / 2$. Therefore, the sole values of $\beta$ that lead to standard electric charges for these fermions are $\beta= \pm 1 / \sqrt{3}$ [20, 21]. Anomaly cancellation requires that two of the quark families lie in the $\mathbf{3}$, while the remaining family, together with the leptons, lies in the $\overline{\mathbf{3}}$ (or vice versa). Left-handed leptons are accommodated into an $S U(3)_{L}$ triplet, in the same way as the quarks $\mathcal{Q}_{3 L}$, and this can be done with or without the inclusion of nonstandard particles $[8,22,23]$. To get an anomaly-free theory, it is necessary to have the same number of fermions in the $\mathbf{3}$ and the $\overline{\mathbf{3}}$. Therefore, if the model has a quark content as shown in equation (2), the number of lepton families has to be the same as the number of quark colors. In what follows we will not discuss the features of lepton interactions, focusing on the analysis of flavor-changing currents in the quark sector.

The gauge bosons associated with $S U(3)_{L}$ transform according to the adjoint representation of the group, and can be written as

$$
\mathbf{W}_{\mu}=W_{\mu}^{a} \frac{\lambda^{a}}{2}=\frac{1}{2}\left(\begin{array}{ccc}
W_{\mu}^{3}+\frac{1}{\sqrt{3}} W_{\mu}^{8} & \sqrt{2} W_{\mu}^{+} & \sqrt{2} K_{1 \mu} \\
\sqrt{2} W_{\mu}^{-} & -W_{\mu}^{3}+\frac{1}{\sqrt{3}} W_{\mu}^{8} & \sqrt{2} \bar{K}_{2 \mu} \\
\sqrt{2} \bar{K}_{1 \mu} & \sqrt{2} K_{2 \mu} & -\frac{2}{\sqrt{3}} W_{\mu}^{8}
\end{array}\right),
$$

where $\lambda^{a}$ are the Gell-Mann matrices, and the electric charges of $K_{1}$ and $K_{2}$ are given by $Q_{1}=1 / 2+(\sqrt{3} \beta) / 2$ and $Q_{2}=1 / 2-(\sqrt{3} \beta) / 2$, respectively. One has in addition a gauge field $B_{\mu}$ associated with $U(1)_{X}$; this boson is a singlet under $S U(3)_{L}$ and has zero electric charge.

In general, it is convenient to rotate the neutral gauge bosons $W^{3}, W^{8}$ and $B$ into new states $A, Z$ and $Z^{\prime}$, given by

$$
\left(\begin{array}{c}
A \\
Z \\
Z^{\prime}
\end{array}\right)=\left(\begin{array}{ccc}
S_{W} & \beta S_{W} & C_{W} \sqrt{1-\beta^{2} T_{W}^{2}} \\
C_{W} & -\beta S_{W} T_{W} & -S_{W} \sqrt{1-\beta^{2} T_{W}^{2}} \\
0 & -\sqrt{1-\beta^{2} T_{W}^{2}} & \beta T_{W}
\end{array}\right)\left(\begin{array}{c}
W^{3} \\
W^{8} \\
B
\end{array}\right),
$$

where the 'Weinberg angle' $\theta_{W}$ is defined by $T_{W}=\tan \theta_{W}=g^{\prime} / \sqrt{g^{2}+\beta^{2} g^{\prime 2}}, g, g^{\prime}$ being the coupling constants associated with the groups $S U(3)_{L}$ and $U(1)_{X}$, respectively $\left(S_{W}=\sin \theta_{W}\right.$, etc). In the new basis, $A_{\mu}$ (the photon) is the gauge boson corresponding to the generator $Q$, while $Z_{\mu}$ can be identified with the SM $Z$ boson. As in the SM, the extended electroweak symmetry is spontaneously broken in 331 models by the presence of elementary scalars having nonzero vacuum expectation values [24-28]. The symmetry breakdown follows a hierarchy

$$
S U(3)_{L} \otimes U(1)_{X} \stackrel{V}{\longrightarrow} S U(2)_{L} \otimes U(1)_{Y} \stackrel{v}{\longrightarrow} U(1)_{Q}
$$


in which two VEV scales $V$ and $v$, with $V \gg v$, are introduced. The photon is kept as the only massless gauge boson, while the remaining neutral gauge bosons get mixed. In this way, $Z$ and $Z^{\prime}$ turn out to be only approximate mass eigenstates.

In the following we will mainly focus on the models with $\beta= \pm 1 / \sqrt{3}$, in which there are no quarks with exotic charges and the possible existence of fermion and gauge boson mixing and FCNC is maximized. These theories include the so-called 'minimal' [20, 26] and 'economical' [27, 28] 331 models, for which different phenomenological analyses have been carried out in the last few years. The case of 331 models with $\beta= \pm \sqrt{3}$ [1] is briefly addressed in section 4 .

\section{Fermion couplings to neutral gauge bosons}

We are interested in the analysis of neutral currents driven by gauge bosons in the framework of 331 models with $\beta= \pm 1 / \sqrt{3}$. Taking into account the quark content in equation (2), it is easy to obtain the corresponding interaction Lagrangian for the $Z$ and $Z^{\prime}$ gauge bosons. One gets

$$
\begin{aligned}
\mathcal{L}_{\mathrm{NC}}^{\left(Z, Z^{\prime}\right)}=-\frac{g}{2 C_{W}} & \left\{\bar{U}^{0} \gamma_{\mu}\left[\left(C_{W}^{2}-\frac{S_{W}^{2}}{3}\right) P_{L}-\frac{4 S_{W}^{2}}{3} P_{R}\right] U^{0}-\sum_{i=1}^{n_{T}} \frac{4 S_{W}^{2}}{3} \bar{T}_{i}^{0} \gamma_{\mu} T_{i}^{0}\right. \\
& \left.+\bar{D}^{0} \gamma_{\mu}\left[\left(-C_{W}^{2}-\frac{S_{W}^{2}}{3}\right) P_{L}+\frac{2 S_{W}^{2}}{3} P_{R}\right] D^{0}+\sum_{i=1}^{n_{B}} \frac{2 S_{W}^{2}}{3} \bar{B}_{i}^{0} \gamma_{\mu} B_{i}^{0}\right\} Z^{\mu} \\
& -\frac{g}{2 C_{W}} \frac{1}{\sqrt{3 C_{W}^{2}-S_{W}^{2}}}\left\{\bar{U}^{0} \gamma_{\mu}\left[\left(\Lambda C_{W}^{2} \pm \frac{S_{W}^{2}}{3}\right) P_{L} \pm \frac{4 S_{W}^{2}}{3} P_{R}\right] U^{0}\right. \\
& \pm \sum_{i=1}^{n_{T}} \bar{T}_{i}^{0} \gamma_{\mu}\left[\left(-2 C_{W}^{2}+\frac{4 S_{W}^{2}}{3}\right) P_{L}+\frac{4 S_{W}^{2}}{3} P_{R}\right] T_{i}^{0} \\
& +\bar{D}^{0} \gamma_{\mu}\left[\left(C_{W}^{2} \Lambda \pm \frac{S_{W}^{2}}{3}\right) P_{L} \mp \frac{2 S_{W}^{2}}{3} P_{R}\right] D^{0} \\
& \left. \pm \sum_{i=1}^{n_{B}} \bar{B}_{i}^{0} \gamma_{\mu}\left[\left(2 C_{W}^{2}-\frac{2 S_{W}^{2}}{3}\right) P_{L}-\frac{2 S_{W}^{2}}{3} P_{R}\right] B_{i}^{0}\right\} Z^{\prime \mu}
\end{aligned}
$$

where $P_{L, R}=\left(1 \mp \gamma_{5}\right) / 2$, and we have defined the matrices $D^{0}=\left(\begin{array}{lll}d_{1}^{0} & d_{2}^{0} & d_{3}^{0}\end{array}\right)^{T}, U^{0}=$ $\left(\begin{array}{lll}u_{1}^{0} & u_{2}^{0} & u_{3}^{0}\end{array}\right)^{T}$ and $\Lambda=\operatorname{diag}(1,1,-1)$ (flavor space). Superindices 0 indicate that quarks are in the weak interaction basis. Quarks $J$ in equation (2) have been renamed by $T_{i}$ and $B_{i}$ to emphasize that they carry the same electric charges as ordinary top and bottom. The number of these exotic quarks, $n_{T}$ and $n_{B}$, can be 1 or 2 depending on the choice of the parameter $\beta$ : for $\beta=1 / \sqrt{3}$ one has two up-type quarks $T_{1,2}$ (then $n_{T}=2$ ) and one down-type quark $B_{1}\left(n_{B}=1\right)$, while for $\beta=-1 / \sqrt{3}$ one has $n_{T}=1, n_{B}=2$. Due to the particular representation structure of the quark sector, the interactions between the $Z^{\prime}$ boson and the left-handed quarks include the matrix $\Lambda$, which is not proportional to the identity. This leads to tree-level FCNC after rotating the quarks to the mass eigenstate basis. In addition, note that there is no universality between the interactions of ordinary and exotic left-handed quarks, both for $Z$ and $Z^{\prime}$ currents. In this way, the mixing between ordinary and exotic quarks leads to further sources of FCNC.

It is noteworthy that for $\beta= \pm 1 / \sqrt{3}$ the gauge bosons $K_{1}$ and $K_{2}$ will have electric charges 0 or 1 . Therefore, in general they will mix with the remaining neutral gauge bosons 
and the $W^{+}$. For $\beta=1 / \sqrt{3}$ one gets a neutral gauge boson $K_{2}^{0}$, and the corresponding weak neutral current reads

$$
\mathcal{L}_{\mathrm{NC}}^{\left(K_{2}\right)}=-\frac{g}{\sqrt{2}}\left(\bar{B}_{1}^{0} \gamma_{\mu} P_{L} d_{3}^{0}+\sum_{i=1}^{2} \bar{u}_{i}^{0} \gamma_{\mu} P_{L} T_{i}^{0}\right) K_{2}^{\mu}+\text { h.c. },
$$

while for $\beta=-1 / \sqrt{3}$, the neutral state is $K_{1}$, leading to the neutral current

$$
\mathcal{L}_{\mathrm{NC}}^{\left(K_{1}\right)}=-\frac{g}{\sqrt{2}}\left(\bar{u}_{3}^{0} \gamma_{\mu} P_{L} T_{1}^{0}-\sum_{i=1}^{2} \bar{B}_{i}^{0} \gamma_{\mu} P_{L} d_{i}^{0}\right) K_{1}^{\mu}+\text { h.c. }
$$

Let us take for definiteness $\beta=1 / \sqrt{3}$. As one can see from equation (3), the complex fields $K_{2}$ and $\bar{K}_{2}$ are in fact combinations of the neutral gauge bosons $W^{6}$ and $W^{7}$. These are naturally defined as $\mathrm{CP}$-even and $\mathrm{CP}$-odd states, respectively, since these definitions lead to a $\mathrm{CP}$-invariant weak current in the interaction basis. Thus, once the photon has been identified, the remaining four neutral gauge boson mass eigenstates will be written in general as linear combinations of $Z, Z^{\prime}, W^{6}$ and $W^{7}$, and the mixing between $W^{7}$ and any of the other will imply nonconservation of the $\mathrm{CP}$ symmetry in the theory. An equivalent reasoning applies to $K_{1}, \bar{K}_{1}, W^{4}$ and $W^{5}$ in the case $\beta=-1 / \sqrt{3}$.

Now it is convenient to organize the nonstandard fermions together with the up- and down-like quarks $U_{i}^{0}$ and $D_{i}^{0}$ into four or five-component fermion vectors $\mathcal{U}, \mathcal{D}$ defined as

$$
\mathcal{U}^{0}=\left(\begin{array}{c}
U^{0} \\
T^{0}
\end{array}\right), \quad \mathcal{D}^{0}=\left(\begin{array}{c}
D^{0} \\
B^{0}
\end{array}\right)
$$

where $T^{0}=\left(T_{1}^{0} T_{2}^{0}\right)^{T}, B^{0}=B_{1}^{0}$ for $\beta=1 / \sqrt{3}$ and $T^{0}=T_{1}^{0}, B^{0}=\left(B_{1}^{0} B_{2}^{0}\right)^{T}$ for $\beta=-1 / \sqrt{3}$. With this notation the neutral currents driven by the $Z$ and $Z^{\prime}$ bosons can be written as

$$
\begin{aligned}
\mathcal{L}_{\mathrm{NC}}^{\left(Z, Z^{\prime}\right)}=-\frac{g}{2 C_{W}} \sum_{f=\mathcal{U}, \mathcal{D}}\left[\bar{f}^{0} \gamma^{\mu}\left(\epsilon_{f_{L}}^{(1)} P_{L}+\epsilon_{f_{R}}^{(1)} P_{R}\right) f^{0} Z_{\mu}\right. \\
\left.+\frac{1}{\sqrt{3 C_{W}^{2}-S_{W}^{2}}} \bar{f}^{0} \gamma^{\mu}\left(\epsilon_{f_{L}}^{(2)} P_{L}+\epsilon_{f_{R}}^{(2)} P_{R}\right) f^{0} Z_{\mu}^{\prime}\right],
\end{aligned}
$$

where

$$
\begin{aligned}
\epsilon_{\mathcal{U}_{L}}^{(1)}= & \left(C_{W}^{2}-\frac{S_{W}^{2}}{3}\right) \mathbb{1}-\left(\begin{array}{ll}
0_{3 \times 3} & \\
& \mathbb{1}_{n_{T} \times n_{T}}
\end{array}\right), \\
\epsilon_{\mathcal{D}_{L}}^{(1)}= & \left(-C_{W}^{2}-\frac{4 S_{W}^{2}}{3}\right) \mathbb{1}+\left(\begin{array}{ll}
0_{3 \times 3} & \\
& \mathbb{1}_{n_{B} \times n_{B}}
\end{array}\right) \\
\epsilon_{\mathcal{U}_{R}}^{(1)}= & -\frac{4 S_{W}^{2}}{3} \mathbb{1}, \quad \epsilon_{\mathcal{D}_{R}}^{(1)}=\frac{2 S_{W}^{2}}{3} \mathbb{1} \\
\epsilon_{\mathcal{U}_{L}}^{(2)}= & \left(C_{W}^{2} \pm \frac{S_{W}^{2}}{3}\right) \mathbb{1}-2 C_{W}^{2}\left(\begin{array}{ll}
0_{2 \times 2} & \mathbb{1}_{\left(n_{T}+1\right) \times\left(n_{T}+1\right)}
\end{array}\right) \\
& \quad+\left(C_{W}^{2} \mp 2 C_{W}^{2} \pm S_{W}^{2}\right)\left(\begin{array}{cc}
0_{3 \times 3} & \mathbb{1}_{n_{T} \times n_{T}}
\end{array}\right) \\
\epsilon_{\mathcal{D}_{L}}^{(2)}= & \left(C_{W}^{2} \pm\right. \\
& \left.\frac{S_{W}^{2}}{3}\right) \mathbb{1}-2 C_{W}^{2}\left(\begin{array}{ll}
0_{2 \times 2} & \\
\mathbb{1}_{\left(n_{B}+1\right) \times\left(n_{B}+1\right)}
\end{array}\right) \\
& \quad+\left(C_{W}^{2} \pm 2 C_{W}^{2} \mp S_{W}^{2}\right)\left(\begin{array}{cc}
0_{3 \times 3} & \mathbb{1}_{n_{B} \times n_{B}}
\end{array}\right)
\end{aligned}
$$


$\epsilon_{\mathcal{U}_{R}}^{(2)}= \pm \frac{4 S_{W}^{2}}{3} \mathbb{1}, \quad \epsilon_{\mathcal{D}_{R}}^{(2)}=\mp \frac{2 S_{W}^{2}}{3} \mathbb{1}$

(blank entries in the above matrices are zeros). In the same way the neutral currents involving $K$ vector bosons for $\beta=1 / \sqrt{3}$ are given by

$\mathcal{L}_{\mathrm{NC}}^{(K)}=-\frac{g}{\sqrt{2}} \sum_{f=\mathcal{U}, \mathcal{D}}\left(\bar{f}^{0} \gamma^{\mu} \epsilon_{f_{L}}^{(3)} P_{L} f^{0} \operatorname{Re} K_{2 \mu}+\mathrm{i} \bar{f}^{0} \gamma^{\mu} \epsilon_{f_{L}}^{(4)} P_{L} f^{0} \operatorname{Im} K_{2 \mu}\right)$

with

$\epsilon_{\mathcal{U}_{L}}^{(3)}=\left(\begin{array}{lll}0_{2 \times 2} & & \mathbb{1}_{2 \times 2} \\ & 0 & \\ \mathbb{1}_{2 \times 2} & & 0_{2 \times 2}\end{array}\right), \quad \epsilon_{\mathcal{D}_{L}}^{(3)}=\left(\begin{array}{ccc}0_{2 \times 2} & & \\ & 0 & 1 \\ & 1 & 0\end{array}\right)$,

$\epsilon_{\mathcal{U}_{L}}^{(4)}=\left(\begin{array}{ccc}0_{2 \times 2} & & \mathbb{1}_{2 \times 2} \\ & 0 & \\ -\mathbb{1}_{2 \times 2} & & 0_{2 \times 2}\end{array}\right), \quad \epsilon_{\mathcal{D}_{L}}^{(4)}=\left(\begin{array}{ccc}0_{2 \times 2} & & \\ & 0 & -1 \\ & 1 & 0\end{array}\right)$,

while for $\beta=-1 / \sqrt{3}$, replacing $K_{2} \rightarrow K_{1}$ in equation (16), one has

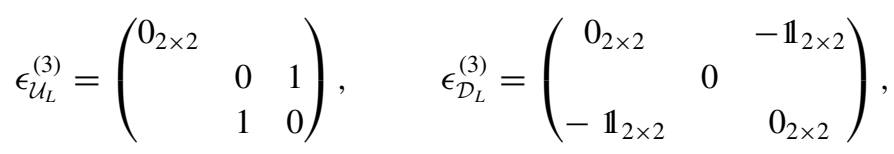

$\epsilon_{\mathcal{U}_{L}}^{(4)}=\left(\begin{array}{ccc}0_{2 \times 2} & & \\ & 0 & 1 \\ & -1 & 0\end{array}\right), \quad \epsilon_{\mathcal{D}_{L}}^{(4)}=\left(\begin{array}{ccc}0_{2 \times 2} & & \mathbb{1}_{2 \times 2} \\ & 0 & \\ -\mathbb{1}_{2 \times 2} & & 0_{2 \times 2}\end{array}\right)$.

Up to now the currents have been written in the interaction basis. In order to move to the fermion mass eigenstate basis, we introduce unitary matrices $V_{L, R}^{f}$, with $f=\mathcal{U}$, $\mathcal{D}$, that diagonalize the fermion mass matrices arising from the Yukawa couplings in the 331 model [26-29]. One has thus

$$
\mathcal{U}_{L, R}^{0}=V_{L, R}^{\mathcal{U}} \mathcal{U}_{L, R}, \quad \mathcal{D}_{L, R}^{0}=V_{L, R}^{\mathcal{D}} \mathcal{D}_{L, R},
$$

where $\mathcal{U}, \mathcal{D}$ are quark mass eigenstate vectors. The matrices $V_{L, R}^{f}$ will have dimensions $4 \times 4$ or $5 \times 5$ for the up or down sector, depending on the choice of the parameter $\beta$. Given the structure of the couplings, it is useful to write the matrices $V_{L}^{\mathcal{U}, \mathcal{D}}$ in terms of submatrices. For $V_{L}^{\mathcal{U}}$ we define

$$
V_{L}^{\mathcal{U}}=\left(\begin{array}{ccc}
V_{q q(2 \times 2)}^{\mathcal{U}} & V_{q Q_{(2 \times 1)}}^{\mathcal{U}} & V_{q J\left(2 \times n_{T}\right)}^{\mathcal{U}} \\
V_{Q q(1 \times 2)}^{\mathcal{U}} & V_{Q Q}^{\mathcal{U}} & V_{Q J\left(1 \times n_{T}\right)}^{\mathcal{U}} \\
V_{J q_{\left(n_{T} \times 2\right)}^{\mathcal{U}}}^{\mathcal{U}} & V_{J Q_{\left(n_{T} \times 1\right)}^{\mathcal{U}}}^{\mathcal{U}} & V_{J J\left(n_{T} \times n_{T}\right)}^{\mathcal{U}}
\end{array}\right)
$$

while the same definitions apply to $V_{L}^{\mathcal{D}}$, with the replacements $\mathcal{U} \rightarrow \mathcal{D}, n_{T} \rightarrow n_{B}$. The textures of these matrices arise from the scalar VEVs and the parameters entering the Yukawa sector of the model. Even if these are in principle unknown, the common belief is that there is a hierarchy in the masses and mixing angles between the quark families. Thus for the moment we will take $V_{L, R}^{f}$ as unknown, and then we will consider the presence of hierarchies between the different submatrices in $V_{L}^{\mathcal{U}, \mathcal{D}}$. Since right-handed currents are flavor diagonal (as occurs in the SM), the mixing matrices $V_{R}^{\mathcal{U}, \mathcal{D}}$ are not observable in 331 models. In contrast, the parameters in $V_{L}^{\mathcal{U}}$ and $V_{L}^{\mathcal{D}}$ become separately observable due to the non-universality of the couplings in equations (10) and (16). 
The charged currents involving the $W^{ \pm}$gauge bosons are similar to those in the SM, i.e.

$\mathcal{L}_{\mathrm{CC}}^{W}=-\frac{g}{\sqrt{2}} \bar{U}^{0} \gamma^{\mu} P_{L} D^{0} W_{\mu}^{+}+$h.c. $=-\frac{g}{\sqrt{2}} \overline{\mathcal{U}}^{0} \gamma^{\mu} P_{L} \epsilon_{W} \mathcal{D}^{0} W_{\mu}^{+}+$h.c.,

where

$$
\epsilon_{W}=\left(\begin{array}{cc}
\mathbb{1}_{3 \times 3} & \\
& 0_{n_{T} \times n_{B}}
\end{array}\right) .
$$

After rotating to the fermion mass eigenstate basis, in principle all quark states interact with the $W^{ \pm}$bosons. We consider in particular the charged currents involving just the ordinary quarks, which can be written as

$$
\mathcal{L}_{\mathrm{CC}}^{(W, \text { ord })}=-\frac{g}{\sqrt{2}} \bar{U} \gamma^{\mu} P_{L} V_{C K M} D W_{\mu}^{+}+\text {h.c. }
$$

where $V_{C K M}$ is the Cabibbo-Kobayashi-Maskawa matrix. In terms of the above-defined submatrices, the latter is given by

$$
V_{C K M}=V_{0}^{\mathcal{U} \dagger} V_{0}^{\mathcal{D}}
$$

with

$$
V_{0}^{f}=\left(\begin{array}{cc}
V_{q q_{(2 \times 2)}^{f}}^{f} & V_{q Q_{(2 \times 1)}^{f}}^{f} \\
V_{Q q_{(1 \times 2)}^{f}}^{f} & V_{Q Q}^{f}
\end{array}\right)
$$

Since $V_{0}^{\mathcal{U}, \mathcal{D}}$ are $3 \times 3$ submatrices of $V_{L}^{\mathcal{U}, \mathcal{D}}$, it is seen that in 331 models with $\beta= \pm 1 / \sqrt{3}$, the CKM matrix is in general not unitary.

In addition one has to take into account the mixing between gauge bosons. We will concentrate in the neutral sector, since the analysis of FCNC is the main purpose of this work. For the moment let us assume that the VEVs of the scalar states in the model can be taken to be real. This assumption depends on the structure of the scalar potential, and means that there is no spontaneous violation of the CP symmetry. If this is the case, the state $\sqrt{2} \operatorname{Im} K$ decouples, becoming an exact mass eigenstate. However, the vector bosons $Z, Z^{\prime}$ and $\sqrt{2} \operatorname{Re} K$ in general get mixed [21, 26-28]. One can rotate to the mass eigenstate basis, say $Z_{1}, Z_{2}, Z_{3}$ (where $Z_{1}$ is the ordinary gauge boson seen in high-energy experiments) through an orthogonal mixing matrix $R$ :

$$
\left(\begin{array}{c}
Z \\
Z^{\prime} \\
\sqrt{2} \operatorname{Re} K
\end{array}\right)=R\left(\begin{array}{l}
Z_{1} \\
Z_{2} \\
Z_{3}
\end{array}\right)
$$

Thus in the fermion and gauge boson mass basis, the neutral currents will be given by

$$
\begin{aligned}
& \mathcal{L}_{\mathrm{NC}}=-\sum_{f=\mathcal{U}, \mathcal{D}} {\left[Q_{f} \bar{f} \gamma^{\mu} f A_{\mu}+\sum_{j, k=1}^{3} g_{j} \bar{f} \gamma^{\mu}\left(E_{f_{L}}^{(j)} P_{L}+E_{f_{R}}^{(j)} P_{R}\right) f R_{j k} Z_{k \mu}\right.} \\
&\left.+\mathrm{i} \frac{g}{2} \bar{f} \gamma^{\mu}\left(E_{f_{L}}^{(4)} P_{L}+E_{f_{R}}^{(4)} P_{R}\right) f \sqrt{2} \operatorname{Im} K_{\mu}\right]
\end{aligned}
$$

where $Q_{f}$ stand for the fermion electric charges, the couplings $g_{j}$ are defined as

$$
g_{1}=\frac{g}{2 C_{W}}, \quad g_{2}=\frac{g^{\prime}}{2 \sqrt{3} S_{W} C_{W}}=\frac{g}{2 \sqrt{3} C_{W} \sqrt{C_{W}^{2}-\beta^{2} S_{W}^{2}}}, \quad g_{3}=\frac{g}{2},
$$

and the flavor matrices $E_{f_{L, R}}^{(j)}$ are given by

$$
E_{f_{L}}^{(i)}=V_{L}^{f \dagger} \epsilon_{f_{L}}^{(i)} V_{L}^{f}, \quad E_{f_{R}}^{(i)}=V_{R}^{f \dagger} \epsilon_{f_{R}}^{(i)} V_{R}^{f}=\epsilon_{f_{R}}^{(i)} .
$$


According to the hierarchy followed by the gauge symmetry breakdown (see equation (5)), it is reasonable to expect that $Z_{2}, Z_{3}, T$ and $B$ get masses of order $V$. These states should decouple for large $V$; hence, the mixing angles between $Z$ and $Z^{\prime}$, Re $K$ and between ordinary and exotic quarks are expected to be small. Explicit calculations for definite scalar potentials and Yukawa couplings can be found in $[4,27,28]$. The mixing angles between light and heavy neutral gauge bosons are typically suppressed by the ratio $(v / V)^{2} \approx\left(m_{Z_{1}} / m_{Z_{2,3}}\right)^{2}$ [4, 28]; hence, one expects mixing angles of order $\lesssim 0.01$ for masses of exotic neutral gauge bosons in the $\mathrm{TeV}$ range.

Let us concentrate on the FCNC currents that involve the ordinary quarks $u, c, t, d, s, b$. It is natural to expect that the presence of these FCNC at the tree level will lead to stringent bounds on the model parameters, and at the same time to the most promising observable effects of new physics at the presently available energy scales. Typically the most stringent bounds for FCNC arise from mass splittings in the neutral meson sectors; therefore, we consider the application of our formalism to these observables in section 4. Other flavor-violating processes that have been experimentally measured/constrained with relatively high precision are e.g. $Z$ boson decays into lepton pairs, quark and lepton radiative decays, and leptonic and semileptonic decays of neutral mesons.

In our framework, tree-level FCNC involving ordinary quarks will arise from the upper left $3 \times 3$ submatrices of the left-handed flavor matrices $E_{f L}^{(i)}$, which are in general not diagonal. For $i=1,2$ we can also separate a diagonal part, given by the first terms of $\epsilon_{f_{L}}^{(i)}$ in equations (11), (13) and (14), which remain unchanged under the fermion state rotation. Thus we write

$$
E_{f_{L}}^{(i)}=E_{f_{L}}^{(i, \mathrm{diag})}+\Delta E_{f_{L}}^{(i)}
$$

With the definitions introduced in equations (22) and (27), the upper-left $3 \times 3$ submatrices of $\Delta E_{f_{L}}^{(i)}$ are given by

$$
\begin{aligned}
\Delta E_{\mathcal{U}_{L}, 3 \times 3}^{(1)}= & -\left(\begin{array}{ll}
V_{J q}^{\mathcal{U} \dagger} V_{J q}^{\mathcal{U}} & V_{J q}^{\mathcal{U} \dagger} V_{J Q}^{\mathcal{U}} \\
V_{J Q}^{\mathcal{U} \dagger} V_{J q}^{\mathcal{U}} & V_{J Q}^{\mathcal{U} \dagger} V_{J Q}^{\mathcal{U}}
\end{array}\right)=-\left(\mathbb{1}_{3 \times 3}-V_{0}^{\mathcal{U} \dagger} V_{0}^{\mathcal{U}}\right) \\
\Delta E_{\mathcal{D}_{L}, 3 \times 3}^{(1)}= & \left(\begin{array}{cc}
V_{J q}^{\mathcal{D} \dagger} V_{J q}^{\mathcal{D}} & V_{J q}^{\mathcal{D} \dagger} V_{J Q}^{\mathcal{D}} \\
V_{J Q}^{\mathcal{D} \dagger} V_{J q}^{\mathcal{D}} & V_{J Q}^{\mathcal{D} \dagger} V_{J Q}^{\mathcal{D}}
\end{array}\right)=\mathbb{1}_{3 \times 3}-V_{0}^{\mathcal{D} \dagger} V_{0}^{\mathcal{D}} \\
\Delta E_{\mathcal{U}_{L}, 3 \times 3}^{(2)}= & -2 C_{W}^{2}\left(\begin{array}{cc}
V_{J q}^{\mathcal{U} \dagger} V_{J q}^{\mathcal{U}}+V_{Q q}^{\mathcal{U} \dagger} V_{Q q}^{\mathcal{U}} & V_{J q}^{\mathcal{U} \dagger} V_{J Q}^{\mathcal{U}}+V_{Q q}^{\mathcal{U} \dagger} V_{Q Q}^{\mathcal{U}} \\
V_{J Q}^{\mathcal{U} \dagger} V_{J q}^{\mathcal{U}}+V_{Q Q}^{\mathcal{U} *} V_{Q q}^{\mathcal{U}} & V_{J Q}^{\mathcal{U} \dagger} V_{J Q}^{\mathcal{U}}+V_{Q Q}^{\mathcal{U} *} V_{Q Q}^{\mathcal{U}}
\end{array}\right) \\
& +\left(C_{W}^{2} \mp 2 C_{W}^{2} \pm S_{W}^{2}\right)\left(\mathbb{1}_{3 \times 3}-V_{0}^{\mathcal{U} \dagger} V_{0}^{\mathcal{U}}\right) \\
\Delta E_{\mathcal{D}_{L}, 3 \times 3}^{(2)}= & -2 C_{W}^{2}\left(\begin{array}{cc}
V_{J q}^{\mathcal{D} \dagger} V_{J q}^{\mathcal{D}}+V_{Q q}^{\mathcal{D} \dagger} V_{Q q}^{\mathcal{D}} & V_{J q}^{\mathcal{D} \dagger} V_{J Q}^{\mathcal{D}}+V_{Q q}^{\mathcal{D} \dagger} V_{Q Q}^{\mathcal{D}} \\
V_{J Q}^{\mathcal{D} \dagger} V_{J q}^{\mathcal{D}}+V_{Q Q}^{\mathcal{D} *} V_{Q q}^{\mathcal{D}} & V_{J Q}^{\mathcal{D} \dagger} V_{J Q}^{\mathcal{D}}+V_{Q Q}^{\mathcal{D} *} V_{Q Q}^{\mathcal{D}}
\end{array}\right) \\
& +\left(C_{W}^{2} \pm 2 C_{W}^{2} \mp S_{W}^{2}\right)\left(\mathbb{1}_{3 \times 3}-V_{0}^{\mathcal{D} \dagger} V_{0}^{\mathcal{D}}\right),
\end{aligned}
$$

where double signs correspond to $\beta= \pm 1 / \sqrt{3}$. We can proceed in a similar way for the FCNC driven by the $K$ vector bosons. In order to have a uniform notation we keep the definition in equation (32), with $E_{f_{L}}^{(i, \text { diag) }}=0$ for $i=3$, 4. Thus for $\beta=1 / \sqrt{3}$ we get

$$
\Delta E_{\mathcal{U}_{L}, 3 \times 3}^{(3)}=\left(\begin{array}{ll}
V_{q q}^{\mathcal{U} \dagger} V_{J q}^{\mathcal{U}}+V_{J q}^{\mathcal{U} \dagger} V_{q q}^{\mathcal{U}} & V_{q q}^{\mathcal{U} \dagger} V_{J Q}^{\mathcal{U}}+V_{J q}^{\mathcal{U} \dagger} V_{q Q}^{\mathcal{U}} \\
V_{q Q}^{\mathcal{U} \dagger} V_{J q}^{\mathcal{U}}+V_{J Q}^{\mathcal{U} \dagger} V_{q q}^{\mathcal{U}} & V_{q Q}^{\mathcal{U} \dagger} V_{J Q}^{\mathcal{U}}+V_{J Q}^{\mathcal{U} \dagger} V_{q Q}^{\mathcal{U}}
\end{array}\right)
$$




$$
\begin{aligned}
& \Delta E_{\mathcal{D}_{L}, 3 \times 3}^{(3)}=\left(\begin{array}{cc}
V_{Q q}^{\mathcal{D} \dagger} V_{J q}^{\mathcal{D}}+V_{J q}^{\mathcal{D} \dagger} V_{Q q}^{\mathcal{D}} & V_{Q q}^{\mathcal{D} \dagger} V_{J Q}^{\mathcal{D}}+V_{J q}^{\mathcal{D} \dagger} V_{Q Q}^{\mathcal{D}} \\
V_{Q Q}^{\mathcal{D} *} V_{J q}^{\mathcal{D}}+V_{J Q}^{\mathcal{D} \dagger} V_{Q q}^{\mathcal{D}} & V_{Q Q}^{\mathcal{D} *} V_{J Q}^{\mathcal{D}}+V_{J Q}^{\mathcal{D} \dagger} V_{Q Q}^{\mathcal{D}}
\end{array}\right) \\
& \Delta E_{\mathcal{U}_{L}, 3 \times 3}^{(4)}=\left(\begin{array}{ll}
V_{q q}^{\mathcal{U} \dagger} V_{J q}^{\mathcal{U}}-V_{J q}^{\mathcal{U} \dagger} V_{q q}^{\mathcal{U}} & V_{q q}^{\mathcal{U} \dagger} V_{J Q}^{\mathcal{U}}-V_{J q}^{\mathcal{U} \dagger} V_{q Q}^{\mathcal{U}} \\
V_{q Q}^{\mathcal{U} \dagger} V_{J q}^{\mathcal{U}}-V_{J Q}^{\mathcal{U} \dagger} V_{q q}^{\mathcal{U}} & V_{q Q}^{\mathcal{U} \dagger} V_{J Q}^{\mathcal{U}}-V_{J Q}^{\mathcal{U} \dagger} V_{q Q}^{\mathcal{U}}
\end{array}\right) \\
& \Delta E_{\mathcal{D}_{L}, 3 \times 3}^{(4)}=\left(\begin{array}{ll}
-V_{Q q}^{\mathcal{D} \dagger} V_{J q}^{\mathcal{D}}+V_{J q}^{\mathcal{D} \dagger} V_{Q q}^{\mathcal{D}} & -V_{Q q}^{\mathcal{D} \dagger} V_{J Q}^{\mathcal{D}}+V_{J q}^{\mathcal{D} \dagger} V_{Q Q}^{\mathcal{D}} \\
-V_{Q Q}^{\mathcal{D} *} V_{J q}^{\mathcal{D}}+V_{J Q}^{\mathcal{D} \dagger} V_{Q q}^{\mathcal{D}} & -V_{Q Q}^{\mathcal{D} *} V_{J Q}^{\mathcal{D}}+V_{J Q}^{\mathcal{D} \dagger} V_{Q Q}^{\mathcal{D}}
\end{array}\right),
\end{aligned}
$$

while for $\beta=-1 / \sqrt{3}$, one obtains similar expressions, changing $\Delta E_{\mathcal{U}_{L}, 3 \times 3}^{(3,4)} \rightarrow-\Delta E_{\mathcal{D}_{L}, 3 \times 3}^{(3,4)}$ and $\Delta E_{\mathcal{D}_{L}, 3 \times 3}^{(3,4)} \rightarrow \Delta E_{\mathcal{U}_{L}, 3 \times 3}^{(3,4)}$.

Our results can be compared with those obtained in [17], where the authors study FCNC driven by a $Z^{\prime}$ boson associated with an additional Abelian gauge symmetry. The model in that work does not consider the presence of exotic fermions, nor that of a gauge boson analogous to the $K$; therefore, in general the structure of FCNC is different from that of the 331 models. However, it is easily seen that in the limit where both the mixing between ordinary and exotic quarks and the mixing between $K$ and $Z, Z^{\prime}$ gauge bosons are neglected, our expressions reduce to a particular case of those quoted in [17]. Namely, the matrices $B^{\psi_{L, R}}$ of [17], responsible for flavor-changing effects, are nothing but our flavor matrices $E_{f_{L, R}}^{(2)}$, and the corresponding coupling $g_{2}$ is that given by equation (30). Therefore, in the above-mentioned limit, the phenomenological analysis carried out in [17] is directly applicable to 331 models.

As stated, the elements of the matrices $V_{L}^{f}$ can be obtained from the Yukawa coupling constants and the VEVs of the neutral scalar fields. Since these parameters are not known, in practice the only conditions to be satisfied are the unitarity of $V_{L}^{\mathcal{U}, \mathcal{D}}$ and the experimental constraints on $V_{C K M}=V_{0}^{\mathcal{U} \dagger} V_{0}^{\mathcal{D}}$ (these constraints have to be taken with care, since $V_{C K M}$ is in general not unitary). However, most theoretical models of fermion mass matrices lead to hierarchies in quark mixing angles, which are related to the corresponding hierarchies in quark masses. This is clearly inspired in the experimental measurements of $V_{C K M}$, which is surprisingly close to the identity. According to this picture not only the product $V_{0}^{\mathcal{U}} V_{0}^{\mathcal{D}}$ would be close to the identity but also $V_{0}^{\mathcal{U} \dagger}$ and $V_{0}^{\mathcal{D}}$ separately.

In order to analyze the phenomenological consequences of these mass matrix structures in the context of the 331 theories, let us study the dominant contributions to tree-level FCNC. For definiteness we will consider the case in which off-diagonal elements of mixing matrices satisfy family hierarchies given by

$$
V_{i j}^{f} \sim\left(\frac{m_{f_{i}}}{m_{f_{j}}}\right)^{1 / 2} .
$$

These hierarchies in $V_{i j}^{f}$ are obtained in various models for quark mass matrices, which have been widely studied in the literature [30,31]. Note that the assumption of this ansatz for the mixing submatrices $V_{0}^{\mathcal{U}, \mathcal{D}}$ in the quark basis defined in equation (2) corresponds to a picture of approximate flavor symmetries in which the third family of ordinary quarks is associated with the states $u_{3}$ and $d_{3}$. This is also suggested by the notation in equation (22), where we have labeled with $Q$ and $q$ quarks belonging to the $Q_{3 L}$ and $Q_{1,2 L}$ multiplets, respectively. In fact, the third family could be associated with any combination of $Q_{1 L}, Q_{2 L}$ and $Q_{3 L}$. However, once approximate flavor symmetries are considered, it is natural to assume that the family that distinguishes from the other two (in the sense that the states transform according to a different $S U$ (3) representation) is the third (heavier) family of ordinary quarks. 
It is interesting to note that, within this framework, one can distinguish between FCNC in the up- and down-quark sectors. For the up-quark sector, given the small ratios $m_{u} / m_{t}$ and $m_{c} / m_{t}$, equation (43) implies values of order $\lesssim 10^{-1}$ for the elements of $V_{q Q}^{\mathcal{U}}$ and $V_{Q q}^{\mathcal{U}}$, which mix quarks $u, c$ with quark $t$. Here it is interesting to consider the case in which the $S U(3)_{L} \otimes U(1)_{X}$ breaking scale is of the order of $1 \mathrm{TeV}$. In this case one expects exotic quarks $T$ to have masses of the TeV order; therefore, the elements of $V_{Q J}^{\mathcal{U}}$ and $V_{J Q}^{\mathcal{U}}$ can be relatively large. In this way, one has

$$
\begin{aligned}
& \Delta E_{\mathcal{U}_{L}, 3 \times 3}^{(1)} \simeq-\left(\begin{array}{cc}
V_{J q}^{\mathcal{U} \dagger} V_{J q}^{\mathcal{U}} & V_{J q}^{\mathcal{U} \dagger} V_{J Q}^{\mathcal{U}} \\
V_{J Q}^{\mathcal{U} \dagger} V_{J q}^{\mathcal{U}} & 1-\left|V_{Q Q}^{\mathcal{U}}\right|^{2}
\end{array}\right) \\
& \Delta E_{\mathcal{U}_{L}, 3 \times 3}^{(2)} \simeq-2 C_{W}^{2}\left(\begin{array}{cc}
V_{Q q}^{\mathcal{U} \dagger} V_{Q q}^{\mathcal{U}} & V_{Q q}^{\mathcal{U} \dagger} V_{Q Q}^{\mathcal{U}} \\
V_{Q Q}^{\mathcal{U} *} V_{Q q}^{\mathcal{U}} & 1
\end{array}\right)+\left(C_{W}^{2} \mp 2 C_{W}^{2} \pm S_{W}^{2}\right)\left(\begin{array}{cc}
0_{2 \times 2} & 0_{2 \times 1} \\
0_{1 \times 2} & 1-\left|V_{Q Q}^{\mathcal{U}}\right|^{2}
\end{array}\right),
\end{aligned}
$$

where we have used the relation $V_{J Q}^{\mathcal{U} \dagger} V_{J Q}^{\mathcal{U}}+\left|V_{Q Q}^{\mathcal{U}}\right|^{2}=1-V_{q Q}^{\mathcal{U} \dagger} V_{q Q}^{\mathcal{U}} \simeq 1$. Equations (44) and (45) show that $Z$ and $Z^{\prime}$-mediated FCNC in the up-quark sector are expected to be suppressed by the small values of $V_{J q}^{\mathcal{U}}$ and $V_{Q q}^{\mathcal{U}}$, respectively.

On the other hand, in the down-quark sector, it is natural to expect $m_{b} \ll m_{B}$; therefore, ordinary quarks decouple, and both $q J$ and $Q J$ mixing will be suppressed. The submatrix $V_{0}^{\mathcal{D}}$ can be taken in this case as approximately unitary. However, one can expect a significant mixing between $b$ and $s$ quarks $\left(\sqrt{\left(m_{s} / m_{b}\right)} \simeq 0.15\right)$, leading to non-negligible values of the matrix elements of $V_{q Q}$ and $V_{Q q}$. One has then

$$
\begin{aligned}
& \Delta E_{\mathcal{D}_{L}, 3 \times 3}^{(1)}=\mathbb{1}_{3 \times 3}-V_{0}^{\mathcal{D} \dagger} V_{0}^{\mathcal{D}} \\
& \Delta E_{\mathcal{D}_{L}, 3 \times 3}^{(2)} \simeq-2 C_{W}^{2}\left(\begin{array}{cc}
V_{Q q}^{\mathcal{D} \dagger} V_{Q q}^{\mathcal{D}} & V_{Q q}^{\mathcal{D} \dagger} V_{Q Q}^{\mathcal{D}} \\
V_{Q Q}^{\mathcal{D} *} V_{Q q}^{\mathcal{D}} & V_{Q Q}^{\mathcal{D} *} V_{Q Q}^{\mathcal{D}}
\end{array}\right) .
\end{aligned}
$$

Since, as stated, $V_{0}^{\mathcal{D}}$ is approximately unitary, it is seen that $Z$-mediated FCNC should be suppressed. On the other hand, the off-diagonal matrix elements in (47) could be significant enough so that the size of $Z^{\prime}$-mediated FCNC in the down sector could be comparable with (one-loop) SM contributions.

Note that the effect of $Q J$ mixing in the up-quark sector should be taken into account when considering the unitarity of the $V_{C K M}$ matrix. Indeed, neglecting $q Q, q J$ mixing in the up-quark sector, as well as $q J, Q J$ mixing in the down-quark sector, one finds

$$
V_{C K M}^{\dagger} V_{C K M}=\left(\begin{array}{ll}
V_{0}^{\mathcal{U} \dagger} V_{0}^{\mathcal{D}}
\end{array}\right)^{\dagger} V_{0}^{\mathcal{U} \dagger} V_{0}^{\mathcal{D}} \simeq\left(\begin{array}{cc}
\mathbb{1}_{2 \times 2} & \\
& 1-V_{J Q}^{\mathcal{U} \dagger} V_{J Q}^{\mathcal{U}}
\end{array}\right) .
$$

This deviation from unitarity could be a signature for the presence of new physics. In particular, note that equation (48) implies $\left|V_{u b}\right|^{2}+\left|V_{c b}\right|^{2}+\left|V_{t b}\right|^{2}<1$, which could be tested from direct measurements of the corresponding matrix elements. This test would require the analysis of the decay $t \rightarrow W b$, which could be achieved in forthcoming experiments such as the LHC.

Finally, from equations (39)-(42), one can determine the dominant contributions to treelevel FCNC mediated by $K$ vector bosons. For $\beta=1 / \sqrt{3}$, one has

$$
\Delta E_{\mathcal{U}_{L}, 3 \times 3}^{(3)} \simeq\left(\begin{array}{ll} 
& V_{J Q}^{\mathcal{U}} \\
V_{J Q}^{\mathcal{U} \dagger} &
\end{array}\right), \quad \Delta E_{\mathcal{U}_{L}, 3 \times 3}^{(4)} \simeq\left(\begin{array}{ll}
V_{J Q}^{\mathcal{U}} \\
-V_{J Q}^{\mathcal{U} \dagger}
\end{array}\right)
$$


while for $\beta=-1 / \sqrt{3}$ we get

$$
\Delta E_{\mathcal{U}_{L}, 3 \times 3}^{(3)} \simeq\left(\begin{array}{ll}
0_{2 \times 2} & \\
& 2 \operatorname{Re}\left(V_{Q Q}^{\mathcal{U} *} V_{J Q}^{\mathcal{U}}\right)
\end{array}\right), \quad \Delta E_{\mathcal{U}_{L}, 3 \times 3}^{(4)} \simeq\left(\begin{array}{ll}
0_{2 \times 2} & \\
& -2 \mathrm{i} \operatorname{Im}\left(V_{Q Q}^{\mathcal{U} *} V_{J Q}^{\mathcal{U}}\right)
\end{array}\right)
$$

For both values of $\beta$, the elements of $\Delta E_{\mathcal{D}_{L, 3 \times 3}}^{(3,4)}$ will be strongly suppressed, according to the above assumptions on Yukawa coupling hierarchies. From the above equations it is seen that only in the up-quark sector and for $\beta=1 / \sqrt{3}$ one would expect sizable $K$-mediated FCNC. Though the disentanglement of the $K$ contribution from the $Z$ and $Z^{\prime}$ ones could be challenging, it is noticeable that it would offer the opportunity to distinguish between models with different choices of $\beta$ without the direct observation of exotic quarks or leptons.

\section{Neutral meson mass differences}

As an application of the formalism presented above, let us analyze the effective Lagrangian for $\Delta F=2$ four-quark couplings ( $F$ here for flavor) arising from the above-described FCNC. This effective interaction is particularly important since $\Delta F=2$ observables, such as neutral meson mass differences in the $K, B$ and $D$ sectors, provide stringent bounds for FCNC $[9,11,12]$.

If we denote the flavor eigenstates of a neutral meson system by $P^{0}$ and $\bar{P}^{0}$, and the corresponding mass eigenstates by $P_{1}$ and $P_{2}$, the mass difference $\Delta m_{P}=m_{P_{1}}-m_{P_{2}}$ will be given by

$$
\Delta m_{P}=\frac{\operatorname{Re}\left\langle P^{0}\left|\mathcal{H}_{\mathrm{eff}}^{(\Delta F=2)}\right| \bar{P}^{0}\right\rangle}{m_{P}} .
$$

With the notation introduced in the previous sections, the effective Lagrangian in 331 models can be written as

$\mathcal{L}_{\text {eff }}^{(\Delta F=2)}=-\frac{4 G_{F}}{\sqrt{2}} \sum_{f=\mathcal{U}, \mathcal{D}} \sum_{a \neq b} C_{a b}^{(f)} \bar{f}_{a} \gamma^{\mu} P_{L} f_{b} \bar{f}_{a} \gamma^{\mu} P_{L} f_{b}$,

where $a, b=1,2,3$ and

$C_{a b}^{(f)}=\sum_{j, k, l=1}^{3}\left[\frac{g_{j} g_{l}}{g^{2}}\left(\Delta E_{f_{L}}^{(j)}\right)_{a b}\left(\Delta E_{f_{L}}^{(l)}\right)_{a b} R_{j k} R_{l k} \frac{m_{W}^{2}}{m_{Z_{k}}^{2}}\right]-\frac{m_{W}^{2}}{4 m_{Z_{k}}^{2}}\left[\left(\Delta E_{f_{L}}^{(4)}\right)_{a b}\right]^{2}$. by

In this way, the contribution from tree-level FCNC to the $P_{1}-P_{2}$ mass difference is given

$$
\Delta m_{P}=\frac{4 \sqrt{2}}{3} G_{F} m_{P} F_{P}^{2} B_{P} \operatorname{Re}\left[C_{a b}^{(f)}\right]
$$

where $f$ is $\mathcal{U}$ or $\mathcal{D}$ depending on the flavor content of $P$, and $B_{P}$ parametrizes the theoretical uncertainty in the evaluation of the hadronic matrix elements. Within the vacuum insertion approximation one has $B_{P}=1$.

Now it is interesting to consider the effective Lagrangian in equations (52) and (53) to distinguish between different 331 scenarios.

- Although we have concentrated in 331 models with $\beta= \pm 1 / \sqrt{3}$, it is also interesting to analyze the models with $\beta= \pm \sqrt{3}$ [1]. The latter (which, actually, were proposed firstly in the literature) include exotic quarks that carry exotic electric charges, namely $5 / 3$ and $-4 / 3$; therefore, they do not mix with ordinary quarks. Moreover, there are no neutral 
$K$ vector bosons. Since $V_{L}^{\mathcal{U}, \mathcal{D}}$ reduce to $V_{0}^{\mathcal{U}, \mathcal{D}}$, which are now unitary, there is no FCNC mediated by the $Z$ boson. Equation (53) reduces in this case to

$$
C_{a b}^{(f)}=\frac{m_{W}^{2}}{4 C_{W}^{2}}\left(\frac{g_{2}}{g_{1}}\right)^{2}\left[\left(\Delta E_{f_{L}}^{(2)}\right)_{a b}\right]^{2}\left(\frac{\sin ^{2} \theta}{m_{Z_{1}}^{2}}+\frac{\cos ^{2} \theta}{m_{Z_{2}}^{2}}\right),
$$

where

$$
\Delta E_{f_{L}}^{(2)}=-2 C_{W}^{2}\left(\begin{array}{cc}
V_{Q q}^{f \dagger} V_{Q q}^{f} & V_{Q q}^{f \dagger} V_{Q Q}^{f} \\
V_{Q Q}^{f *} V_{Q q}^{f} & V_{Q Q}^{f *} V_{Q Q}^{f}
\end{array}\right)
$$

and $\theta$ is the mixing angle in the $2 \times 2$ matrix $R$ that mixes $Z$ and $Z^{\prime}$ states $\left(R_{12}=\right.$ $\left.-R_{21}=\sin \theta\right)$. As it is shown e.g. in [32], this angle is suppressed by the VEV scale ratio $(v / V)^{2}$. Bounds for 331 model parameters have been determined in this context in [11] from various FCNC observables. It is also important to note that the expression in equation (55) can be obtained from the analysis in [17], applied to the case of the 331 model as discussed in the previous section.

In addition, it is noteworthy that

$$
\left(\frac{g_{2}}{g_{1}}\right)^{2}=\frac{1}{3\left(C_{W}^{2}-\beta^{2} S_{W}^{2}\right)}
$$

hence, for $\beta= \pm \sqrt{3}$, the ratio is divergent for $S_{W}^{2}=1 / 4$, where one finds a Landau-like pole for the coupling $g^{\prime}$. If one requires the interaction to be perturbative, the presence of this pole imposes in general an upper bound of a few $\mathrm{TeV}$ for the symmetry breaking scale $V$ [33] (this bound can be somewhat evaded, however, by enlarging the particle content of the model [34]). Models with $\beta= \pm 1 / \sqrt{3}$ are free from this constraint. A brief comparative analysis of FCNC in the $B$ meson sector for different choices of $\beta$ can be found in [10].

- Let us consider 331 models with $\beta= \pm 1 / \sqrt{3}$, assuming a quark-mixing hierarchy such as that in equation (43). As stated above, in this case $Z$ - and $K$-mediated FCNC in the down-quark sector are negligible. One has then

$$
C_{a b}^{(\mathcal{D})} \simeq \frac{m_{W}^{2}}{4 C_{W}^{2}\left(3 C_{W}^{2}-S_{W}^{2}\right)}\left[\left(\Delta E_{\mathcal{D}_{L}}^{(2)}\right)_{a b}\right]^{2} \sum_{k=1}^{3} \frac{R_{2 k}^{2}}{m_{Z_{k}}^{2}} .
$$

Moreover, in general one expects the mixing between $Z$ and $Z^{\prime}$ states, i.e. $R_{21}$, to be of order $(v / V)^{2}$; therefore, the dominant effect is due to the contribution of the (heavy) $Z_{2}$ intermediate state. As shown in [10], the stringent bounds for the $Z_{2}$ mass are then provided by the measured values of $\Delta m_{B_{d}}$ and $\Delta m_{B_{s}}$. From the above expressions, taking into account the result in equation (47), one gets

$\Delta m_{B_{q}}^{\left(Z_{2}\right)} \simeq \frac{4 \sqrt{2}}{3} \frac{C_{W}^{2}}{3 C_{W}^{2}-S_{W}^{2}} G_{F} m_{B_{q}} F_{B_{q}}^{2} B_{B_{q}} \frac{m_{q}}{m_{b}}\left(\frac{m_{W}}{m_{Z_{2}}}\right)^{2}, \quad q=d, s$.

The present experimental results for these observables lead to $m_{Z_{2}} \gtrsim 7 \mathrm{TeV}$. Clearly, this bound can be relaxed either if one considers interferences with SM one-loop contributions or other sources of FCNC, or if one relaxes the hierarchy relation in the quark mixing angles. Within the economical 331 model, a general analysis of the constraints for masses and mixing angles from $\Delta F=2$ observables has been carried out in [12].

On the other hand, in the up-quark sector, for $\beta=1 / \sqrt{3}$, the largest FCNC couplings are those driven by the neutral $K$ state, subsequently followed by those driven by the $Z^{\prime}$ and the $Z$. Thus, depending on the gauge boson masses and mixing angles, the contributions of 
$Z_{1}, Z_{2}$ and $Z_{3}$ could be competitive and all terms in (53) should be taken into account. For $\beta=-1 / \sqrt{3}$, given the large $K$ mass, the contribution of the $K$ state is clearly suppressed in comparison with that of the $Z$; therefore $\Delta E_{\mathcal{U}_{L}}^{(3,4)}$ can be neglected, and only $Z$ and $Z^{\prime}$ contributions compete. The presence of tree-level FCNC in the up-quark sector leads to nonstandard contributions to the $\Delta m_{D}$ mass difference, which has been recently measured [35] attracting significant theoretical interest. Considering the quark-mixing hierarchy in equation (43), and neglecting the mixing between neutral gauge boson states, one can obtain a definite prediction for the order of magnitude of the new contributions. From equations (44) and (45), we get

$$
\begin{aligned}
\Delta m_{D}^{\left(Z_{1}\right)} & \simeq \frac{\sqrt{2}}{3} G_{F} m_{D} F_{D}^{2} B_{D} \frac{m_{u} m_{c}}{m_{T}^{2}} \\
\Delta m_{D}^{\left(Z_{2}\right)} & \simeq \frac{\sqrt{2}}{3} G_{F} m_{D} F_{D}^{2} B_{D} \frac{4 C_{W}^{2}}{3 C_{W}^{2}-S_{W}^{2}} \frac{m_{u} m_{c}}{m_{t}^{2}}\left(\frac{m_{W}}{m_{Z_{2}}}\right)^{2}
\end{aligned}
$$

( $K$-mediated FCNC are negligible for this observable). If we consider the upper bound of $7 \mathrm{TeV}$ for $Z_{2}$ suggested by $B_{d}$ and $B_{s}$ mixing, the contribution $\Delta m_{D}^{Z_{2}}$ from the above expression is found to be about two orders of magnitude below the experimental value $\Delta m_{D}^{(\exp )}=(2.4 \pm 0.7) \times 10^{10} \hbar \mathrm{s}^{-1}$. However, the contribution mediated by the ordinary $Z_{1}$ boson could be significantly larger if the mass of the exotic $T$ quark is in the TeV range. Taking $B_{D}=0.82[36]$ and $F_{D}=0.207 \mathrm{GeV}$, one has

$$
\Delta m_{D}^{\left(Z_{1}\right)} \sim 2 \times 10^{9} \hbar \mathrm{s}^{-1}\left(\frac{1 \mathrm{TeV}}{m_{T}}\right)^{2} ;
$$

thus, it is seen that an observable effect could be achieved if the experimental errors are reduced. It is clear that the above result has to be taken just as an indicative order of magnitude. However, this rough calculation illustrates the possible size of the effects one could expect in 331 models with $\beta= \pm 1 / \sqrt{3}$ within the ansatz of quark-mixing hierarchy given by equation (43).

- If spontaneous CP violation is allowed, equation (53) should still be generalized to allow for the mixing between $Z_{1,2,3}$ and $Z_{4}$. This can be implemented in equation (53) by removing the last term, enlarging $R$ to a $4 \times 4$ orthogonal matrix and extending the sum up to four. The entries $R_{i 4}, R_{4 i}$ with $i=1,2,3$ will be responsible for CP violation. These elements will arise from the imaginary parts of scalar VEVs, which are in general nonzero if CP is spontaneously broken together with the gauge symmetry [37]. However, note that if the scalar sector is minimal (i.e. in the case of 'economical' 331 models), VEV phases can be removed through a global gauge transformation. In this case $Z_{4}$ decouples, becoming an exact mass eigenstate.

\section{Summary}

In summary, we present here a general analysis of tree-level flavor-changing neutral currents in the context of 331 models. We quote the corresponding expressions for arbitrary quark and gauge boson mixing matrices, and then analyze the approximate results obtained for a definite texture of the quark mass matrices. Differences between various 331 models are pointed out.

Our analysis generalizes previous results, including FCNC contributions that are usually neglected in the literature, such as those arising from the mixing between ordinary and exotic quarks and the mixing between neutral gauge bosons. Even if these contributions are in most cases expected to be suppressed, it is seen that they can be significant for some observables, such as e.g. the mass difference between neutral $D$ meson states. 


\section{Acknowledgments}

This work has been supported in part by Banco de la República (Colombia) and by CONICET and ANPCyT (Argentina), under grants PIP 6009, PIP 112-200801-02495, PICT04-03-25374 and PICT07-03-00818.

\section{References}

[1] Pisano F and Pleitez V 1992 Phys. Rev. D 46410 Frampton P H 1992 Phys. Rev. Lett. 692889

Foot R, Hernandez O F, Pisano F and Pleitez V 1993 Phys. Rev. D 474158

[2] Ng D 1994 Phys. Rev. D 494805

[3] kiyan T, Maekawa T and Yokoi S 2002 Mod. Phys. Lett. A 171813

[4] Diaz R A, Martinez R and Ochoa F 2005 Phys. Rev. D 72035018

[5] Montero J C, Pires C A de S and Pleitez V 2001 Phys. Lett. B 502167 Montero J C, Pires C A de S and Pleitez V 2002 Phys. Rev. D 65095001

[6] Fregolente D and Tonasse M D 2003 Phys. Lett. B 5557

Hoang L N and Lan N Q 2003 Europhys. Lett. 64571

Filippi S, Ponce W A and Sanchez L A 2006 Europhys. Lett. 73142

Pires C A de S and Silva P S Rodrigues da 2007 J. Cosmol. Astropart. Phys. JCAP12(2007)012

[7] Diaz R A, Gallego D and Martinez R 2007 Int. J. Mod. Phys. A 221849

[8] Liu J T and Ng D 1994 Phys. Rev. D 50548

Liu J T 1994 Phys. Rev. D 50542

[9] Dumm D Gomez, Pisano F and Pleitez V 1994 Mod. Phys. Lett. A 91609

[10] Rodriguez J A and Sher M 2004 Phys. Rev. D 70117702

[11] Promberger C, Schatt S and Schwab F 2007 Phys. Rev. D 75115007

[12] Cabarcas J M, Dumm D Gomez and Martinez R 2008 Phys. Rev. D 77036002

[13] Promberger C, Schatt S, Schwab F and Uhlig S 2008 Phys. Rev. D 77115022

[14] Martinez R and Ochoa F 2008 Phys. Rev. D 77065012

[15] Cabarcas J M, Dumm D Gomez and Martinez R 2008 Eur. Phys. J. C 58569

[16] Dittmar M, Nicollerat A S and Djouadi A 2004 Phys. Lett. B 583111

[17] Langacker P and Plumacher M 2000 Phys. Rev. D 62013006

[18] Nardi E 1993 Phys. Rev. D 481240

[19] Perez M A, Tavares-Velasco G and Toscano J J 2004 Phys. Rev. D 69115004

[20] Foot R, Hoang L N and Tran T A 1994 Phys. Rev. D 5034

Hoang L N 1996 Phys. Rev. D 53437

Hoang L N 1996 Phys. Rev. D 544691

[21] Ozer M 1996 Phys. Rev. D 541143

[22] Foot R, Hernandez O F, Pisano F and Pleitez V 1993 Phys. Rev. D 474158

[23] Montero J C, Pires C A de S and Pleitez V 2002 Phys. Rev. D 66113003

[24] Nguyen T A, Ky N A and Hoang L N 2000 Int. J. Mod. Phys. A 15283

[25] Tully M B and Joshi G C 2003 Int. J. Mod. Phys. A 181573

[26] Diaz R A, Martinez R and Ochoa F 2004 Phys. Rev. D 69095009

[27] Ponce W A, Giraldo Y and Sanchez L A 2003 Phys. Rev. D 67075001

[28] Dong P V, Hoang L N, Nhung D T and Soa D V 2006 Phys. Rev. D 73035004

[29] Cotaescu I I and Palcu A 2008 Mod. Phys. Lett. A 231011

[30] Fritzsch H 1978 Phys. Lett. B 73317 Fritzsch H 1979 Nucl. Phys. B 155189

[31] Cheng T P and Sher M 1987 Phys. Rev. D 353484

[32] Liu J T and Ng D 1994 Z. Phys. C 62693

[33] Dias A G, Martinez R and Pleitez V 2005 Eur. Phys. J. C 39101

[34] Dias A G 2005 Phys. Rev. D 71015009

[35] Aubert B et al (BABAR Collaboration) 2007 Phys. Rev. Lett. 98211802 Staric M et al (BABAR Collaboration) 2007 Phys. Rev. Lett. 98211803

[36] Gupta R, Bhattacharya T and Sharpe S R 1997 Phys. Rev. D 554036

[37] Epele L, Fanchiotti H, Canal C Garcia and Dumm D Gomez 1995 Phys. Lett. B 343291 Dumm D Gomez 1996 Int. J. Mod. Phys. A 11887 